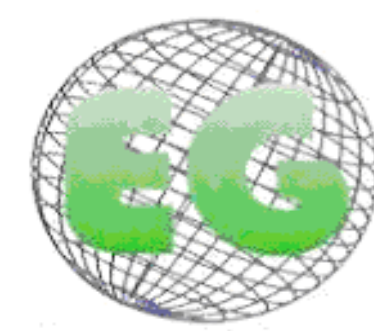

ISSN 1695-6141

$\mathbf{N}^{\circ} 23$
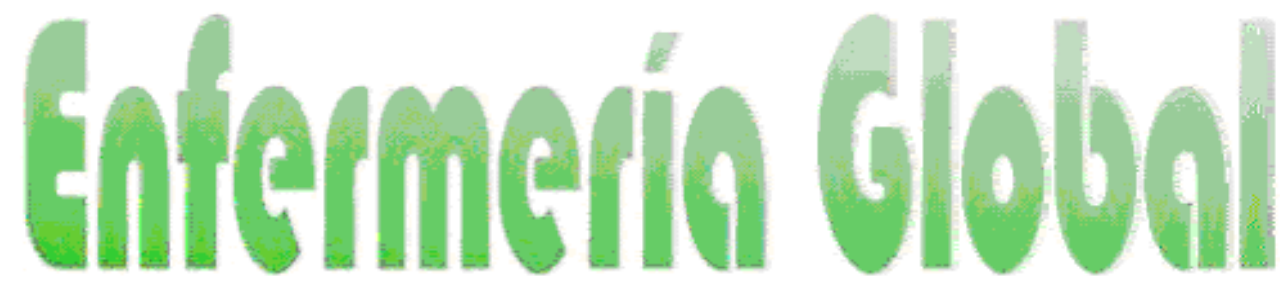

Revista electrónica trimestral de Enfermería

www.um.es/egloball

REVISIONES

\title{
Análisis de satisfacción del paciente quirúrgico en oftalmología.
}

Analysis of surgical patient satisfaction surgical in ophthalmology.

*Minchón Hernando, A., Díaz Jiménez, M., Cutilla Muñoz, MA., De Porras

Carrasco, R., Domínguez García, G., Vázquez de la Rosa, MP.

*DUE. UGC Oftalmología. Área hospitalaria Juan Ramón Jiménez. HUELVA

Palabras clave: Satisfacción de pacientes; Servicios de Enfermería; Calidad; Acceso y Evaluación de la Atención en Salud.

Keywords: Patient satisfaction; Nursing services; Healt Care Quality; Access and Evaluation

\section{RESUMEN}

Objetivos: Conocer la percepción y valoración sobre satisfacción de los pacientes con patología quirúrgica oftálmica intervenida. Identificar puntos débiles y establecer estrategias de mejora organizativa a partir de los resultados obtenidos.

Material y método: Estudio descriptivo, retrospectivo y trasversal. Encuesta elaborada "ad hoc" con 18 preguntas.

Resultados: Los pacientes que contestan el cuestionario autoadministrado presentan una edad media de $70,76 \pm 8,9$ años. Se observa un $59,37 \%$ de hombres frente a un $31,26 \%$ de mujeres con un tiempo medio de espera quirúrgica de 2,2 meses. El $64,52 \%$ de los entrevistados no conoce el nombre de la enfermera.

Conclusiones: Necesidad de poner en marcha de estrategias dirigidas a la mejora de la comunicación con los pacientes-familiares y las enfermeras

\section{ABSTRACT}

Objectives: To understand the perception and assessment of patient satisfaction with surgical pathology operated eyes. Identify weaknesses and develop strategies for organizational improvement from the results.

Material and Methods: Descriptive, retrospective and transversal. Questionnaire conducted with 18 questions.

Results: Patients who answered the questionnaire had a mean age of $70.76 \pm 8.9$ years. There were $59.37 \%$ of men compared with $31.26 \%$ of women with a mean wait of 2.2 months until surgery. $64.52 \%$ of respondents did not know the name of the nurse.

Conclusions: The need to implement strategies aimed at improving communication with patients, families and nurses. 


\section{INTRODUCCIÓN}

La complejidad del sistema sanitario y la amplitud de la oferta de servicios que se requiere para dar respuesta a las necesidades de los pacientes y su entorno, hacen que el valor de la continuidad asistencial y de la visión integrada de la atención tengan un papel cada vez más relevante a la hora de definir o medir la calidad de los servicios que prestamos.

En los últimos tiempos, el paciente se ha convertido en el principal centro e impulso para elevar la calidad de los servicios sanitarios, por lo que su grado de satisfacción es el indicador fundamental de la calidad asistencial ${ }^{1}$. Medir la satisfacción del paciente es un medio de valoración general de los servicios de salud y muy específicamente los servicios prestados por las enfermeras ${ }^{2,3}$. De este modo, verificando de manera válida y contrastada nuestros servicios, podremos generalizar los resultados obtenidos.

Para Deming la calidad significa cumplir con las expectativas del usuario, es el hecho de proporcionarle elementos adecuados a sus necesidades, con una filosofía básica de administración, una nueva forma de trabajar donde prevalece el respeto, la confianza y el trabajo en equipo ${ }^{4}$. Se considera también calidad al conjunto de características de un producto proceso o servicio que le confiere su aptitud para satisfacer las necesidades del cliente o usuario de dichos servicios ${ }^{5}$.

Los servicios sanitarios son de calidad cuando carecen de deficiencia y satisfacen las necesidades del usuario interno y externo. Una asistencia sanitaria de calidad es la aplicación correcta, en la asistencia a los pacientes, de los principios esenciales de las ciencias médicas, al mismo tiempo que se equiparan los riesgos inherentes a cada actividad asistencial realizada a los beneficios que se esperan de ella ${ }^{6}$. Al hablar específicamente de calidad en los centros asistenciales o sanitarios, debemos esperar que se alcance una calidad integral, en la cual se encuentra involucrada la calidad intrínseca, que se refiere a los procesos diagnósticos, terapéuticos o rehabilitadores, al profesionalismo y/o competencia de los prestadores de servicios y a la tecnología disponible para ello y la calidad percibida, valorada fundamentalmente por los usuarios y que es determinada por condicionantes de la satisfacción del usuario, como son: equidad, fiabilidad, efectividad, buen trato, respeto, información, continuidad y confortabilidad.

Desde el Sistema Sanitario Público de Andalucía (SSPA) se reconoce la experiencia del ciudadano como un componente esencial para la mejora de la calidad de los centros y servicios sanitarios. En un sistema sanitario cuya legitimación descansa en la fiabilidad, en la confianza y la satisfacción que generamos entre los ciudadanos y pacientes, la satisfacción es entendida como componente principal de la calidad. Debemos de trabajar para mejorar la salud y aumentar la experiencia positiva de los pacientes en su relación con los servicios ${ }^{7}$. Por todo ello, el SSPA pone en marcha el II Plan de Calidad con tres importantes áreas de actuación, a saber: el ciudadano, los profesionales y, por último, la propia organización sanitaria que integra a ambos ${ }^{8}$.

Desde esta perspectiva de calidad se diseña este estudio donde el objetivo principal es:

$\checkmark$ Conocer la percepción y valoración sobre satisfacción de los pacientes con patología quirúrgica oftálmica intervenidos en los dos hospitales referentes: H. "Juan Ramón Jiménez" y H. "Vázquez Díaz" de Huelva. 
Objetivos secundarios serían:

- Establecer estrategias de mejoras organizativas en base a los resultados obtenidos.

- Identificar puntos débiles relacionados con la calidad asistencial.

\section{MATERIAL Y MÉTODO}

Metodología: Estudio descriptivo, retrospectivo y trasversal.

Población de estudio: Pacientes con patología quirúrgica oftálmica o familiares de los mismos intervenidos de Cirugía Programada con Ingreso, Cirugía Mayor Ambulatoria o cirugía menor ambulatoria (CMA/cma) en los quirófanos de los Hospitales "Juan Ramón Jiménez" y “Vázquez Díaz" de Huelva durante el año 2.010

Muestra: Determinada mediante técnicas de muestreo aleatorio simple para una población ${ }^{9}$ $\mathrm{N}=3.140$ con error estandar (se) de 0,015 e intervalo de confianza del $90 \%$.

$\sigma^{2}=(s e)^{2}=(0,015)^{2}=0,000225$

$s^{2}=p(1-p)=0,9(1-0,9)=0,09$

$n^{\prime}=\frac{s^{2}}{\sigma^{2}}=\frac{0,09}{0,000225}=400$

$n=\frac{n^{n}}{1+\frac{n}{N}}=\frac{400}{1+\frac{400}{\mathrm{r} 140}}=354,92$

; por tanto el tamaño muestral resultante es de 355 cuestionarios cumplimentados para el error estandar e intervalo de confianza descrito.

Criterios de inclusión: Todos los pacientes intervenidos, conscientes y orientados aunque la cumplimentación de la encuesta la realicen los familiares del mismo.

Criterios de exclusión: Pacientes menores de 15 años.

Instrumentos de recolección de datos: Cuestionario de satisfacción realizado "ad hoc" en el año 2009 y pilotado durante el último trimestre de ese año. Presenta preguntas con respuestas dicotómicas, con respuestas valoradas mediante escala de Licker y preguntas abiertas. Análisis de las respuestas mediante estadística descriptiva con hoja de cálculo Excel de Microsoft ${ }^{\circledR}$, distribución de frecuencias, porcentaje de las variables y representación en tablas y gráficos.

Variables de estudio: Persona que contesta el cuestionario, Sexo, Edad de la persona que contesta el cuestionario, Edad del paciente, Nivel de estudios, De qué se ha intervenido, Lugar de intervención, Tiempo transcurrido entre la firma del consentimiento informado y la intervención, Higiene y comodidad de las instalaciones para el paciente, Comodidad de las instalaciones para los familiares, Actitud o disposición de los profesionales para atenderle, Información sanitaria dada por el oftalmólogo antes, después y en el informe de alta; $\mathrm{Ha}$ recibido información de cuidados postquirúrgicos, Conoce el nombre del médico, Conoce el nombre de la enfermera, Confianza, Valoración general de la atención recibida, Qué mejorar, Qué le ha gustado más. 


\section{RESULTADOS}

Los resultados obtenidos del cuestionario autoadministrado se exponen a continuación:

\section{1.- Persona que contesta el cuestionario}

El $62,49 \%$ de los cuestionarios los contesta el paciente frente al $34,38 \%$ que son contestados por los acompañantes. El 3,13\% no refleja este ítem.

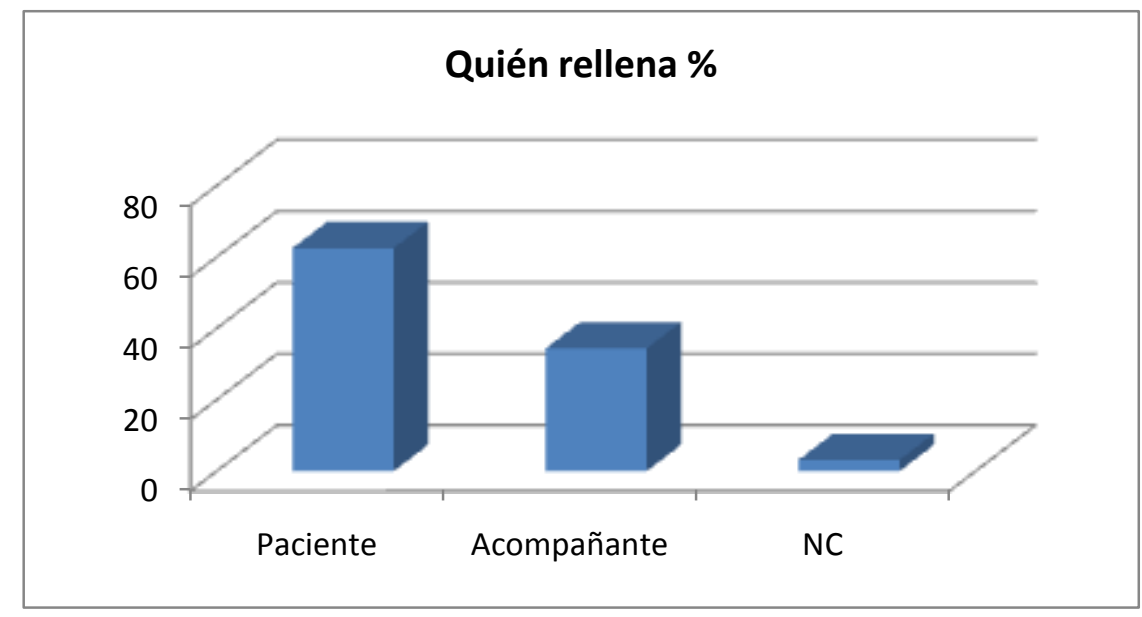

Gráfico no 1: Quién rellena

\section{1.- Sexo}

Los entrevistados son de género masculino en un $59,37 \%$ y de género femenino en un $31,26 \%$. Un $9,37 \%$ de los pacientes no contesta este ítem.

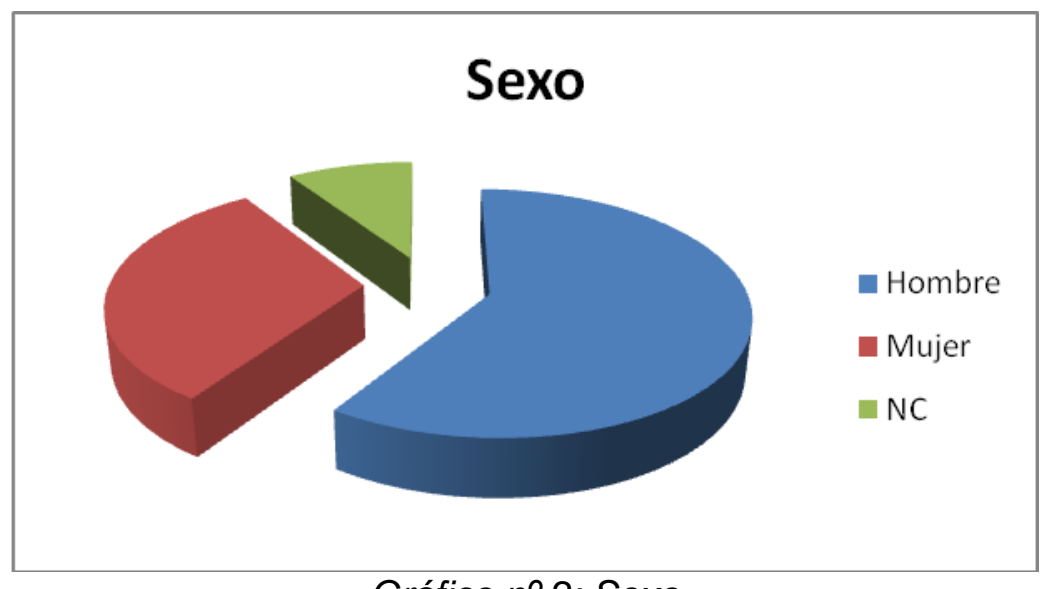

Gráfico nํㅜㄴ: Sexo

\section{2.- Edad de la persona que contesta el cuestionario}

$\begin{array}{ll} & \% \\ \text { +65 años } & 53,125 \\ \text { 45-65 años } & 31,25 \\ \text { 30-45 años } & 15,625 \\ \text { 15-29 años } & 0\end{array}$




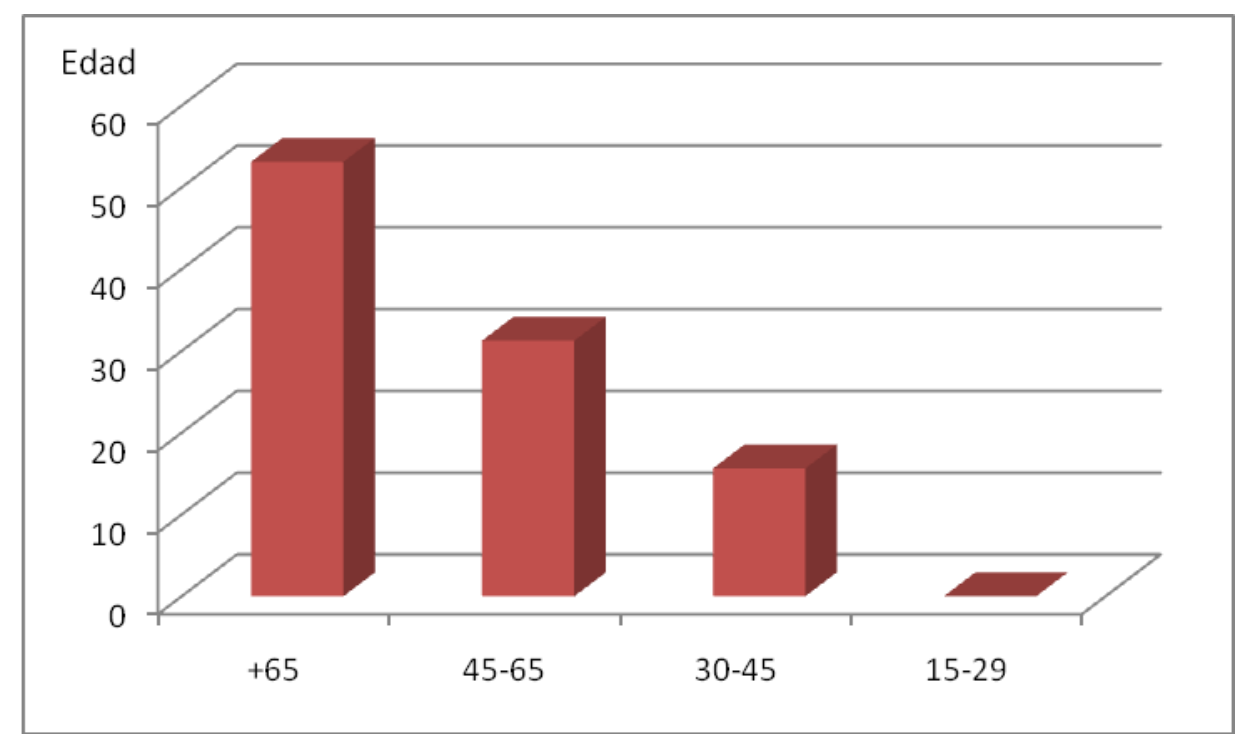

Gráfico nํㅜㄴ Edad de la persona que contesta el cuestionario

\section{3.- Edad del paciente}

Edad media de los pacientes intervenidos: 70,76 \pm 8,9 años

Mediana de edad: 73

\section{4.- Nivel de estudios}

El nivel de estudios de las personas entrevistadas tienes estudios menores de Ed. Primaria en un $34,38 \%$; un $40,63 \%$ con ED. Primaria, un 12,5\% tiene Bachillerato o FP y un $12,5 \%$ tiene estudios universitarios.

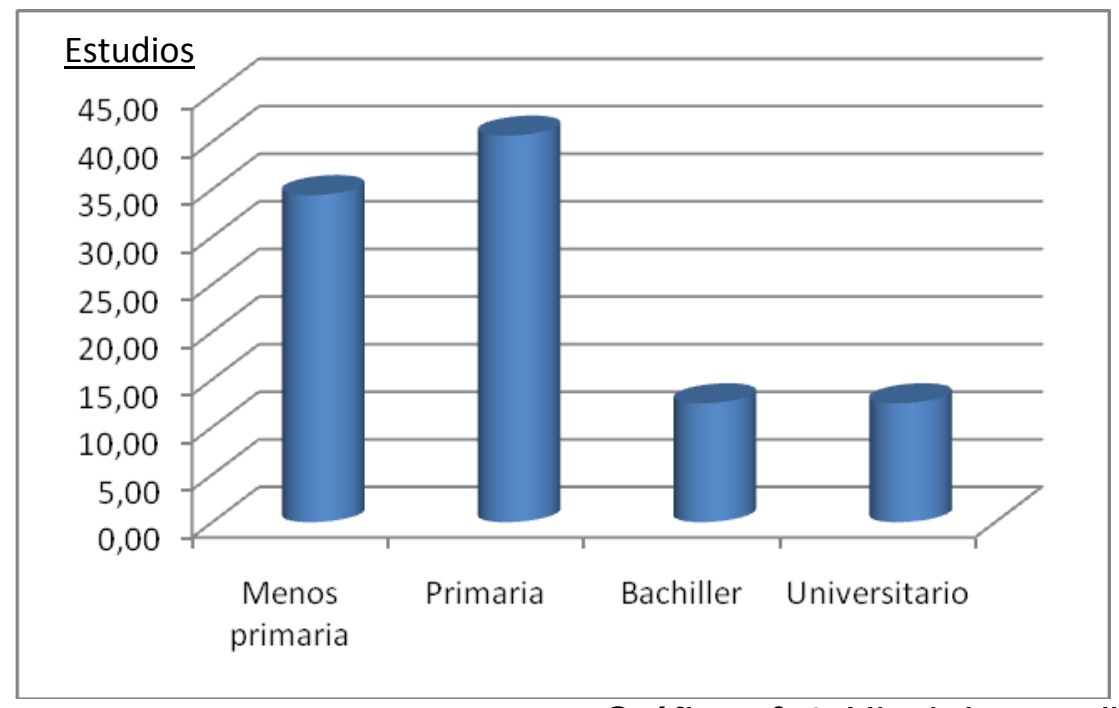

Gráfico nº 4: Nivel de estudios 


\section{5.- De qué se ha intervenido}

En cuanto al tipo de intervención realizada, un $81,25 \%$ es sometido a cirugía de Cataratas, un $6,25 \%$ de Retina, de otros procesos el 9,38 y, por último, un $3,12 \%$ no contesta.

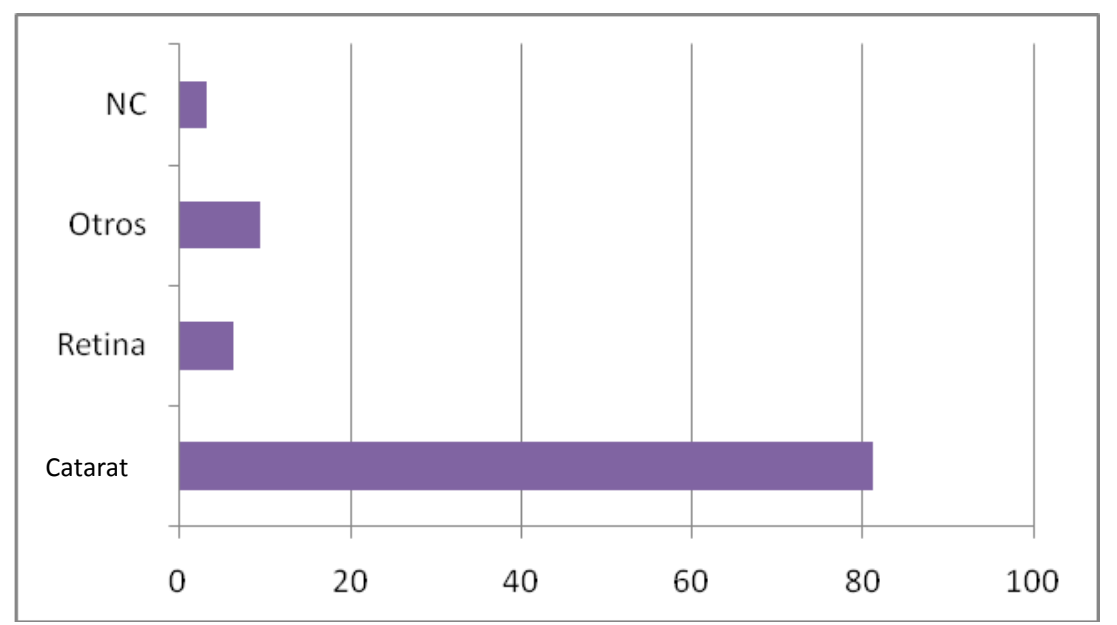

Gráfico nº 5: De que se ha intervenido

\section{6.- Lugar de intervención}

El $56,67 \%$ de los pacientes es intervenido en el hospital "Juan Ramón Jiménez" frente al $43,33 \%$ intervenido en el hospital "Vázquez Díaz".

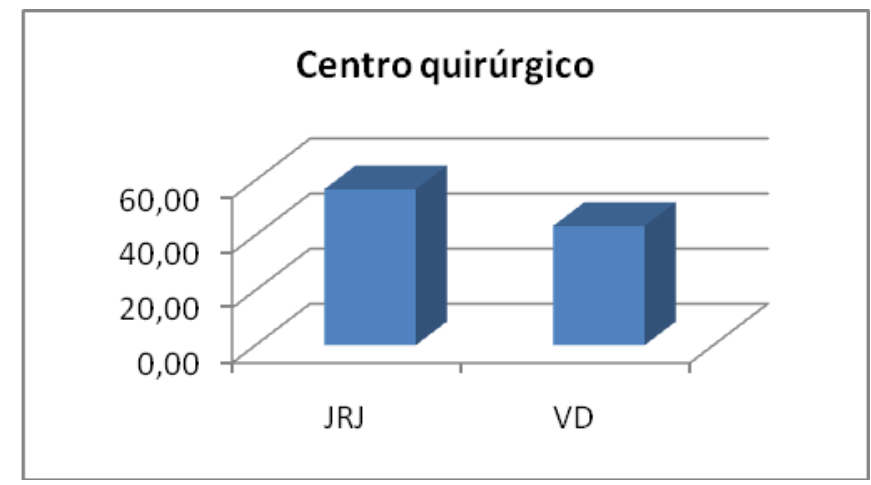

Gráfico $n^{\circ}$ 6: Centro quirúrgico

\section{7.- Tiempo transcurrido desde la firma para la autorización de la cirugía es considerado}

El tiempo transcurrido desde la firma de los Consentimientos Informados $(\mathrm{Cl})$, la inclusión en la Agenda de Gestión de la Demanda (AGD) y la intervención es considerado Muy Bueno (MB) para el $25 \%$ de los usuarios; Bueno (B) para el $40,63 \%$; Regular (R) para el $12,5 \%$; el 9,38\% lo valora como Mal (M); Muy Mal (MM) el 9,38\%. Finalmente, el 3,13\% no contesta (NC).

El tiempo medio de espera es de 2,2 meses desde la firma de la autorización. 


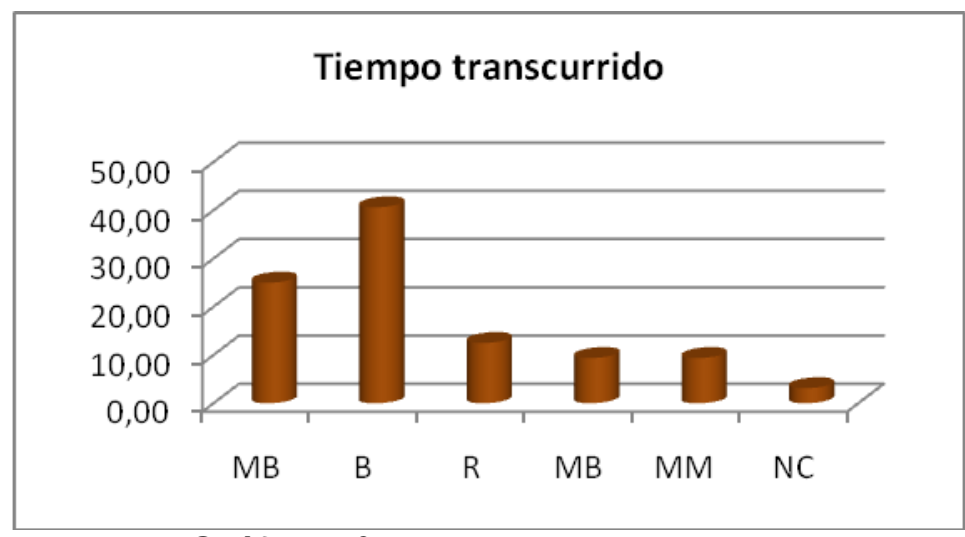

Gráfico no 7: Tiempo transcurrido

\section{8.- Higiene de las instalaciones}

El $100 \%$ de los pacientes opinan que la higiene de las instalaciones está MB ó B

\section{9.- Comodidad de las instalaciones para el paciente}

La comodidad de las instalaciones son consideradas MB por el $37,50 \%$; B por el 46,88\% y $\mathrm{R}$ para el 15,63\%. Ningún paciente las considera M o MM.

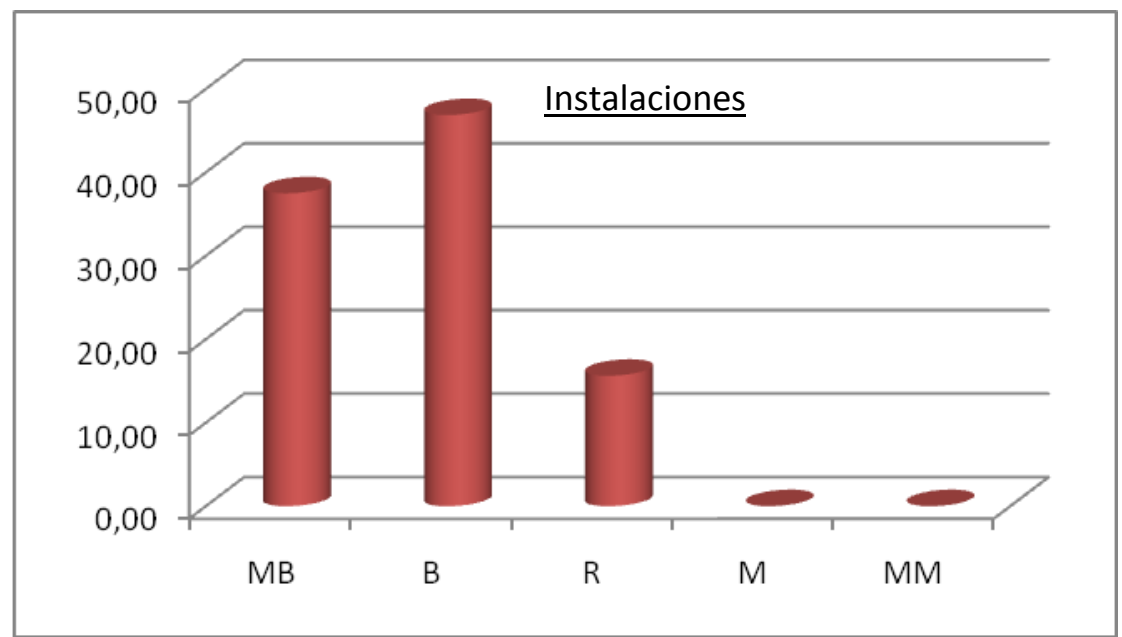

Gráfico nํㅜㅇ: Comodidad instalaciones - Pacientes

\section{0.- Comodidad de las instalaciones para los familiares}

Los familiares consideran la comodidad de las instalaciones MB en un 25\%; B en un $43,75 \%$; $R$ en un $25 \%$ y $M$ en un $6,25 \%$. Ningún acompañante considera las instalaciones MM. 


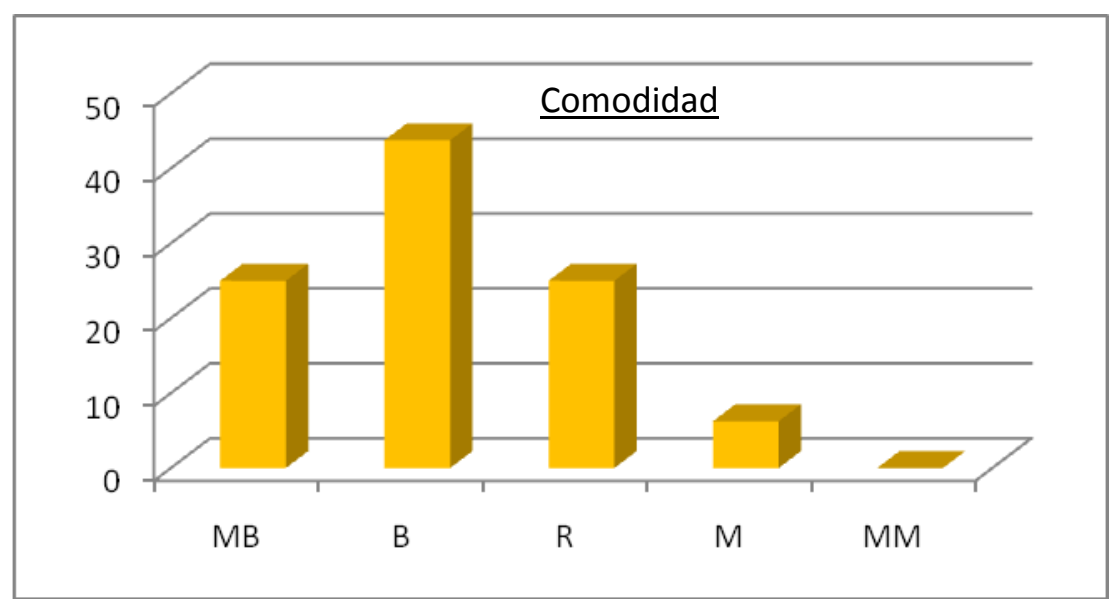

Gráfico no 9: Comodidad instalaciones - familiares

\section{1.- Actitud o disposición del personal para atenderle}

El 100\% de los encuestados opinan que los Médico presentan una actitud MB ó B

Más del $96,88 \%$ de los encuestados opinan que las Enfermeras presentan una actitud MB ó B

1.2, 1.3, 1.4.- Información sanitaria dada por el oftalmólogo antes de la intervención, tras la intervención y en el informe de alta

\begin{tabular}{|l|l|l|l|}
\hline$\%$ & Antes & Después & Info alta \\
\hline MB & 53,13 & 59,38 & 46,88 \\
\hline B & 31,25 & 34,38 & 25,00 \\
\hline R & 12,50 & 3,13 & 12,50 \\
\hline MB & 0,00 & 3,13 & 6,25 \\
\hline MM & 3,13 & 0,00 & 3,13 \\
\hline NC & 0,00 & 0,00 & 6,25 \\
\hline
\end{tabular}

Tabla no I: Información sanitaria

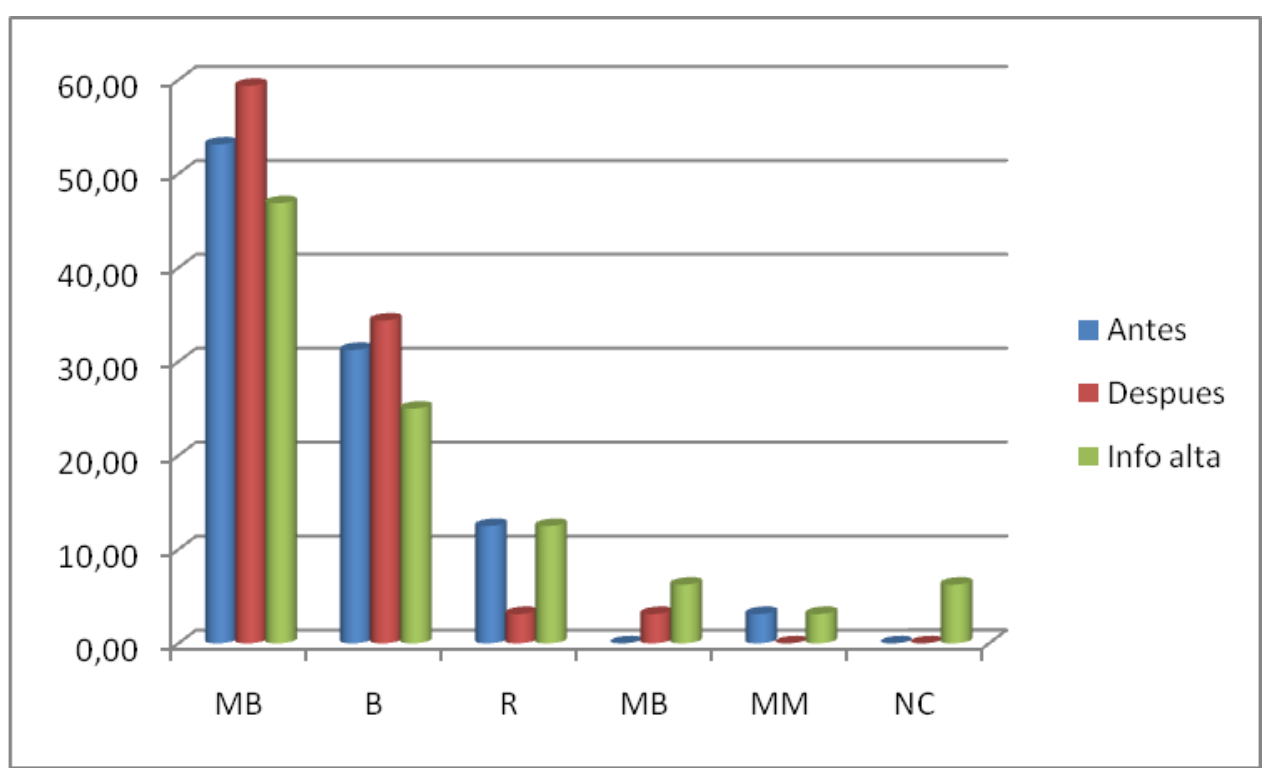

Gráfico nº 10: Información sanitaria 


\section{5.- Ha recibido información de cuidados postquirúrgicos}

El $96,88 \%$ de los pacientes recibe información de cuidados postquirúrgicos. El 3,13 no contesta.

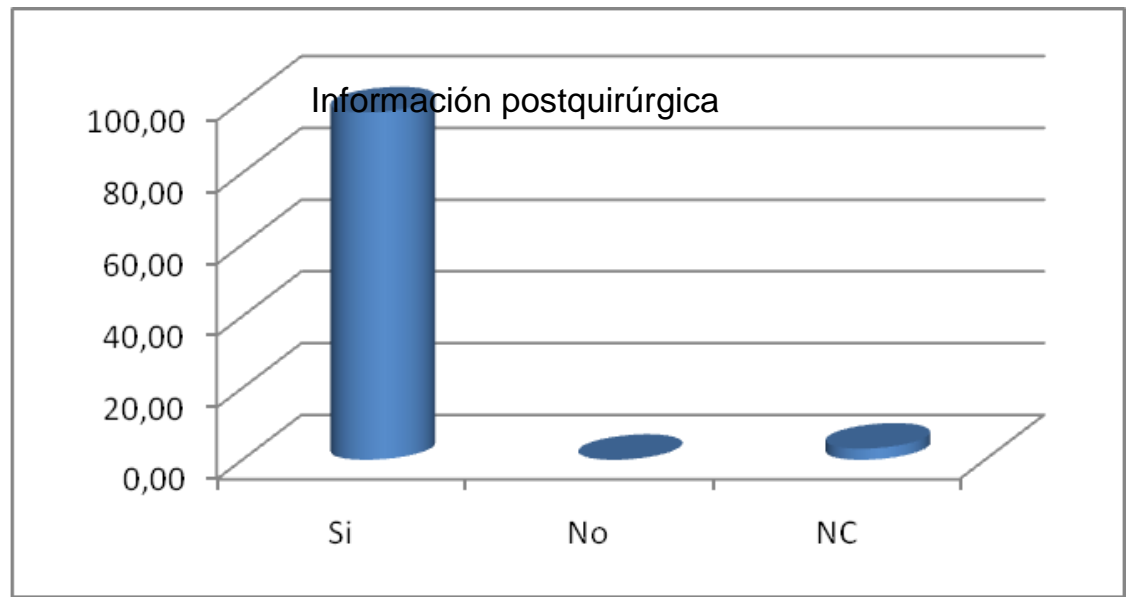

Gráfico nº 11: Cuidados postquirúrgicos

\section{6.- Conoce el nombre del oftalmólogo y de la enfermera}

$\begin{array}{llr}\text { Médico } & \% & \text { Enfermera } \% \\ \mathrm{Si} & 90,63 & 29,03 \\ \mathrm{No} & 6,25 & 64,52 \\ \mathrm{NC} & 3,13 & 6,45\end{array}$

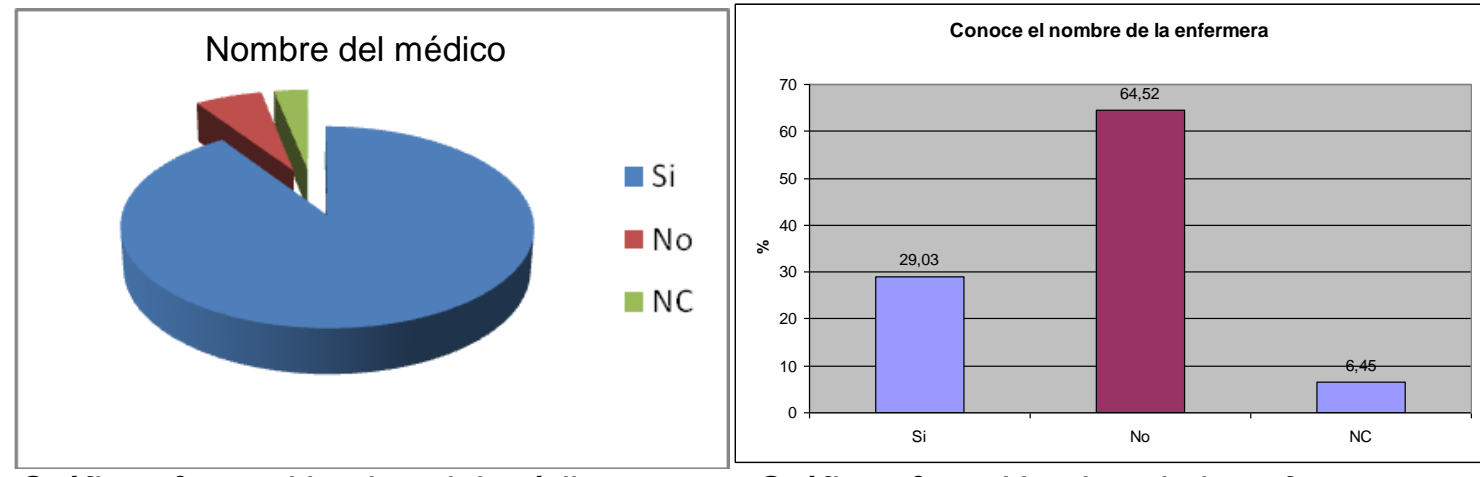

Gráfico nํo 12 : Nombre del médico

Gráfico no 13: Nombre de la enfermera

\section{7.- Son los profesionales dignos de confianza}

Para el $93,75 \%$ de los usuarios, los profesionales sanitarios son dignos de confianza. El $3,13 \%$ considera que no lo son. El 3,13\% restante no contesta. 


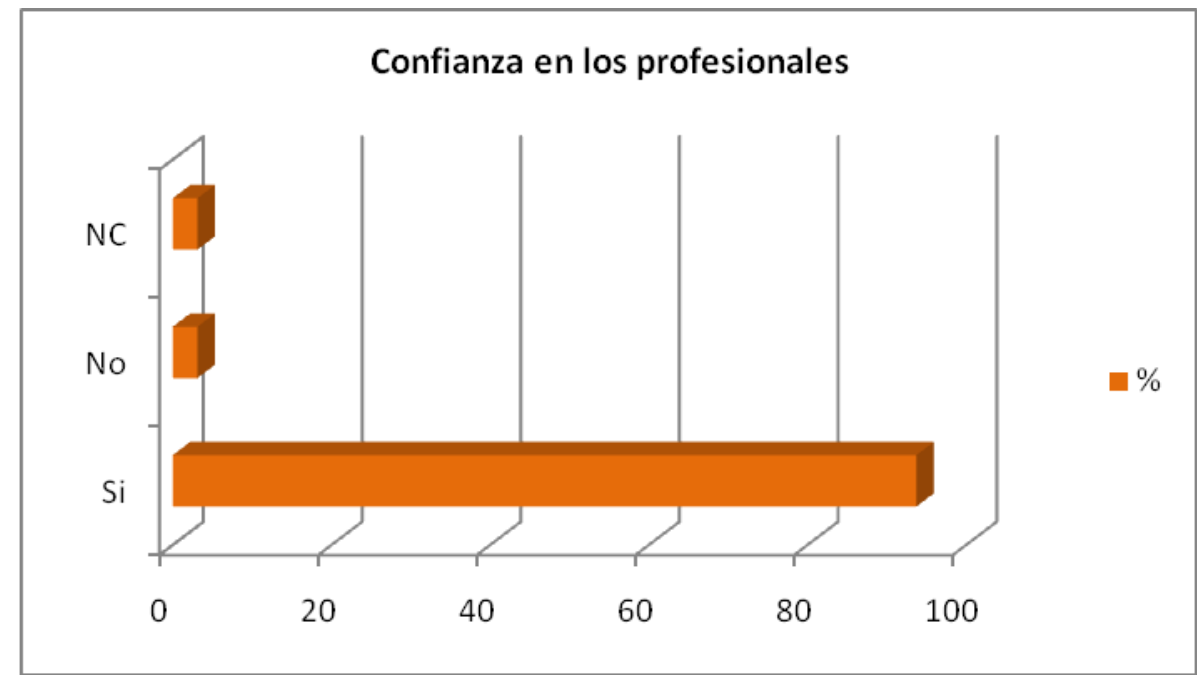

Gráfico no 14: Confianza en los profesionales

\section{8.- Valoración general de la atención recibida}

La atención recibida es considerada MB por el $62,5 \%$; B por el $25 \%$ y $\mathrm{R}$ por $12,5 \%$ restante. Ningún paciente considera la atención recibida M o MM.

\section{DISCUSIÓN}

Los ciudadanos, elemento fundamental sobre el que gira cualquier política de calidad, también de los sistemas sanitarios, deben de ocupar el espacio de participación, autonomía en la toma de decisiones y corresponsabilidad como valores esenciales de la organización sanitaria en particular y de la sociedad en general (Plan de Calidad del SSPA ${ }^{8}$ ). Bajo esta perspectiva de participación y a través del análisis de satisfacción, conocemos la percepción que sobre nuestros servicios de cirugía oftalmológica tienen los usuarios. Si bien es cierto que existen muchos estudios sobre satisfacción de pacientes que resaltan la alta consideración de los cuidados-intervenciones ofrecidas por las enfermeras, en base a nuestros resultados, podemos estar de acuerdo con García Hernandez ${ }^{3}$ en que el carácter educativo de la enfermería es fundamental en adultos mayores para asegurar la comprensión y aumentar la seguridad.

Se han apreciado altos niveles de satisfacción en los ítems correspondiente a la actitud o disposición de los facultativos y enfermeras así como en la confianza depositada en ellos por los propios usuarios, equiparables a resultados obtenidos sobre "calidez de la atención" de estudios consultados ${ }^{3}$.

Por el contrario, si bien los resultados obtenidos en la dimensión del cuidado y en la científico-técnica podemos considerarlos más que satisfactorios; aquellos que tienen que ver con la comodidad de las instalaciones deben ser revisados ya que son un elemento más de la calidad percibida.

\section{CONCLUSIONES}

1. La edad media de los pacientes atendidos es alta, lo que indica la probabilidad de que sean pacientes pluripatológicos, polimedicados, con cierto nivel de dependencia lo que nos orienta a mejorar el vínculo enfermera-paciente en aras de mejorar la información dada al paciente y el consiguiente cumplimiento terapéutico. 
2. Por la misma razón, -el nivel cultural se sitúa en el $71.01 \%$ en estudios primarios o menos que primarios-, hay que asegurar la recepción de la información de manera adecuada por los pacientes.

3. el $31,26 \%$ considera que el tiempo de espera para la intervención tras la firma de $\mathrm{Cl}$ y la inclusión en AGD es considerado Regular o menos, si bien todos los pacientes se han intervenido dentro de los plazos establecidos en el decreto de garantía de plazo del Servicio Andaluz de Salud ${ }^{10}$.

4. Resulta oportuno mejorar las condiciones de la sala de espera quirúrgica donde el $31,25 \%$ de los acompañantes consideran las instalaciones como Regular o menos.

5. La actitud y la información prestada por los profesionales son altamente consideradas así como la confianza que en los mismos depositan los usuarios.

6. Finalmente, destacar que, frente al $90,63 \%$ de los usuarios que conocen el nombre de los facultativos; sólo el $29,03 \%$ de los mismos conoce el nombre de la enfermera.

Parece necesario, a partir de estas conclusiones, la puesta en marcha de estrategias dirigidas a la mejora de la comunicación con los pacientes y familiares, sobre todo desde la enfermería, mejorando la relación enfermera-paciente como parte fundamental de una relación basada en la confianza y seguridad.

\section{BIBLIOGRAFÍA}

1. Díaz R. Satisfacción del paciente: principal motor y centro de los servicios sanitarios. Rev de Calidad Asistencial 2002; 17(1): 22-29.

2. Ramos G MJ. Fiabilidad y validez de un cuestionario de satisfacción del paciente en Guadalajara. Rev Semergen 2000; 26(11): 525-529.

3. García Hernández MD. Satisfacción del usuario adulto mayor con la calidad de la atención de enfermería en el hospital general Cunduacán Tabasco. HORIZONTE SANITARIO 2008; 7(3): 20-30

4. Curso monográfico "Introducción a la calidad de los servicios de enfermería" Paquete didáctico. Instituto Nacional de Enfermedades Respiratorias. México, febrero 2001.

5. Norma española UNE-EN ISO 9001:2008 http://www.faen.es/nueva/Intranet/documentos/2961_ISO9001-2008.pdf Pág visitada el 29 de marzo de 2011

6. Donabedian A. Exploration quality assessment and monitorig, Vol 1. Ann Arbor, Health Administration Press, 1980.

7. Contrato programa. Consejería de Salud. Servicio Andaluz de Salud 2010-2013 http://www.juntadeandalucia.es/salud/export/sites/csalud/galerias/documentos/p_6_p_ 7_contrato_programama/Contrato_Programa_CS_SAS_2010_2013.pdf Pág. visitada el 2 de abril de 2011

8. Plan de Calidad del SSPA. Consejería de Salud de la Junta de Andalucía http://www.juntadeandalucia.es/salud/export/sites/csalud/galerias/documentos/c_1_c_ 6_planes_estrategias/plan_calidad_2010/plan_calidad_2010.pdf Pág visitada el $\overline{7}$ de abril de 2011

9. Sistemas de Información Corporativa. Área Hospitalaria Juan Ramón Jiménez. Servicio Andaluz de Salud. 2010

10. Decreto 209/2001, de 18 de septiembre, por el que se establece la garantía de plazo de respuesta quirúrgica en el Sistema Sanitario Público de Andalucía. BOJA núm. 114 de 2 de octubre 2001 
๑ COPYRIGHT Servicio de Publicaciones - Universidad de Murcia 